\title{
Metabolic Syndrome and Inflammation:
}

\section{A Critical Review of In Vitro and Clinical Approaches for Benefit Assessment of Plant Food Supplements}

\author{
Chiara Di Lorenzo, Mario Dell'Agli, Elisa Colombo, \\ Enrico Sangiovanni, and Patrizia Restani \\ Dipartimento di Scienze Farmacologiche e Biomolecolari, Università degli Studi di Milano, Via Balzaretti 9, 20133 Milan, Italy \\ Correspondence should be addressed to Mario Dell'Agli; mario.dellagli@unimi.it
}

Received 13 December 2012; Revised 21 January 2013; Accepted 21 January 2013

Academic Editor: Ravirajsinh N. Jadeja

Copyright (c) 2013 Chiara Di Lorenzo et al. This is an open access article distributed under the Creative Commons Attribution License, which permits unrestricted use, distribution, and reproduction in any medium, provided the original work is properly cited.

\begin{abstract}
Metabolic syndrome is defined as the clustering in an individual of several metabolic abnormalities associated with insulin resistance, type 2 diabetes, and obesity, in which low-grade chronic inflammatory activity is commonly observed. Part of the European Project PlantLIBRA is concerned with methods to assess the benefits of plant food supplements (PFSs) in countering inflammatory activity and metabolic syndrome. This paper summarizes the current methods used for benefit assessment of PFS, taking into consideration only in vitro, in silico, and clinical methodologies used to investigate the anti-inflammatory properties of plants. No in silico studies (using computer simulation) related to metabolic syndrome were found; these methods appear to be used exclusively for identifying or testing potentially effective compounds in drug development. Most in vitro methods for the assessment of beneficial effects of botanicals or plant food supplements in diabetes were based on a quantitative polymerase chain reaction (PCR), whereas the preferred kind of clinical study was the double-blind randomized controlled clinical trial. Only two parameters were observed to change after treatment with botanicals in both in vitro and in vivo studies: interleukin- 6 and tumour necrosis factor- $\alpha$, and these biomarkers should be carefully considered in future studies for PFS benefit assessment.
\end{abstract}

\section{Introduction}

Metabolic syndrome (MS) defines the clustering in an individual of multiple metabolic abnormalities [1]. World Health Organization and programs including the National Cholesterol Education Program (NCEP) and Adult Treatment Program III (ATP III) have now agreed $[2,3]$ to consider MS as a disease characterized by five traits: (1) increased abdominal girth, (2) low levels of high-density lipoprotein cholesterol (HDL-C), (3) hypertriglyceridemia, (4) hypertension, and (5) fasting hyperglycemia. A low-grade chronic inflammatory activity is commonly observed in metabolic diseases such as obesity and type 2 diabetes (T2D).

A major shortcoming of current definitions of MS is the lack of inclusion of measures of a proinflammatory state and oxidative stress $[4,5]$. The multicenter Insulin Resistance Atherosclerosis Study had shown a linear relation between the inflammatory marker C-reactive protein (CRP) and a number of metabolic disorders [6]. Other proinflammatory markers known to increase in patients with MS include fibrinogen [2], cytokines such as interleukin-6 (IL-6), and tumour necrosis factor- $\alpha$ (TNF- $\alpha$ ).

Type 2 diabetes (T2D) is considered an MS-related disease and an inflammatory disease. As with MS, patients with T2D show higher levels of circulating CRP, fibrinogen, plasminogen activator inhibitor (PAI), and proinflammatory cytokines such as interleukin-1 $\beta$ (IL-1 $\beta$ ) and IL-6. Circulating levels of IL-18 have been reported to be elevated in subjects with the metabolic syndrome and closely associated with the biomarkers of the syndrome to predict cardiovascular events and mortality in populations affected by MS-related diseases [7]. In patients with T2D, metabolic stress promotes insulin resistance and activation of IkB kinase- $\beta$ (IKK $\beta$ ) and JUN $\mathrm{N}$-terminal kinase (JNK), which suggests that these kinases 
have key roles in the pathogenesis of this disease [8]. IKK $\beta$ activates nuclear factor- $\kappa \mathrm{B}$ (NF- $\kappa \mathrm{B})$ which induces expression of NF- $\kappa \mathrm{B}$-dependent genes such as proinflammatory cytokines (e.g., TNF $\alpha$ and IL-1 $\beta$ ), so that the suppression of this transcription factor could reduce metabolic disorders and the complications occurring in diabetes (retinopathy, nephropathy, and neuropathy) [8].

In the last 10 years, the link between inflammation and nutrition has become increasingly apparent [8-10]. It has been shown that excessive macronutrient intake can contribute to the inflammatory response occurring in MS [11], whereas some dietary polyphenols are able to reduce the incidence of MS, including diabetes [12]. Many of the metabolites occurring in plants are now recognized as useful for the maintenance of human health, hence the recommended use of plant food supplements (PFSs). A methodology for the safety assessment of botanicals has been promoted by EFSA (European Food Safety Authority), but major bottlenecks remain in its implementation.

The European Project PlantLIBRA (acronym for plant food supplements: levels of intake, benefit, and risk assessments) aims to promote the safe use of PFS or botanicals and the measurement of the risk/benefit ratio related to their consumption. Part of the project is devoted to the discovery of methods for the evaluation of the benefits of PFS and their application and validation. The first step was to review the evidence for PFS benefit in epidemiological, clinical, and intervention studies, in particular the value of PFS as antiinflammatory agents $[13,14]$.

The aim of the present paper was to identify the in vitro and in vivo methods that can detect a decrease of the inflammatory biomarkers that play a key role in metabolic syndrome and diabetes.

\section{Methods}

2.1. Source and Search Strategy. The following databases were searched electronically to identify relevant articles published up to September 2011: PubMed/Medline, SciFinder Scholar, and Cochrane Library. Search limits were in vitro, in silico, clinical methodologies, and the European languages, without limits of year of publication.

A search strategy was developed for each electronic database using specific medical subject heading (MeSH) terms (e.g., inflammation mediators, $\mathrm{C}$ reactive protein CRP, and metabolic $\mathrm{X}$ syndrome) in addition to relevant text keywords (plant extract, plant preparation, methods, and analytical approaches).

The same MeSH terms were used in the T2D area and in the MS area to search for inflammatory biomarkers and plant extracts, and the specific terms diabetes mellitus, noninsulindependent OR diabetes mellitus, and type II were combined with relevant keywords (plant extract, plant preparation, methods, and analytical approaches).

Titles and abstracts of retrieved citations were first screened to identify publications reporting in vivo methods developed in humans, in vitro and in silico methods used in inflammation conditions related to MS or diabetes. Animal studies were not considered since PlantLIBRA neither uses nor promotes in vivo experiments on animals. Other exclusion criteria were the use of plant ingredients for homeopathy, topical use, aerosol/inhalation, and hygiene products. Reviews, commentaries, and patents were also discarded.

\section{Results and Discussion}

The search by title and abstract retrieved 46 papers for MS and 68 for diabetes. After removal of duplicates and application of the inclusion/exclusion criteria, the total number of papers was 43. Papers were also rejected if they were not in a European language. In silico methods for assessing PFS benefit in MS-related diseases were not found. All the studies selected for diabetes were related to T2D.

3.1. Metabolic Syndrome Studies. Neither in silico nor in vitro studies for assessing inflammation in metabolic syndrome were found. Although diabetes is one of the features of MS, the methods related to diabetes have been considered separately from those relating to MS, because of the complexity of the metabolic syndrome, which includes several alterations of metabolic conditions not specifically associated with diabetes.

3.1.1. In Vivo Methods. Table 1 reports the in vivo methods used in clinical trials. The preferred type of clinical study to evaluate the anti-inflammatory effect of PFS in humans was the double-blind randomized controlled clinical trial.

Several publications reported in the present review used randomization in clinical trials, but in a few cases, randomization was not described in detail or incompletely applied (e.g., no randomization for age or gender). Positive aspects of randomization include the elimination of biases, balanced arms, and the capacity to form the basis for statistical tests.

A method of randomization should be considered appropriate if it allows each study participant to have the same chance of receiving the intervention [22]. Methods of allocation using the date of birth or of admission, hospital numbers, or alternation are not appropriate. Suitable methods for randomization include using a table of random numbers or computer generation. In most studies, the subjects enrolled included people of both sexes. Studies of only male or female subjects do not reflect the whole population and the results are not reliable. Almost all studies reported the number of dropouts, but it was not always clear if this was due to lack of efficacy or due to adverse effects. The analytical method used to quantify inflammatory markers such as CRP, cytokines, malondialdehyde (MDA), and adhesion molecules was reported in most studies. In several cases, methods were well described, and manufacturers' protocols were appropriate. The methods most frequently applied were the ELISA test, immunoturbidimetry, and real-time PCR.

3.1.2. Inflammatory Biomarkers Affected by PFS in Metabolic Syndrome. The inflammatory parameters decreasing after PFS treatment were also reviewed. Although changes after 


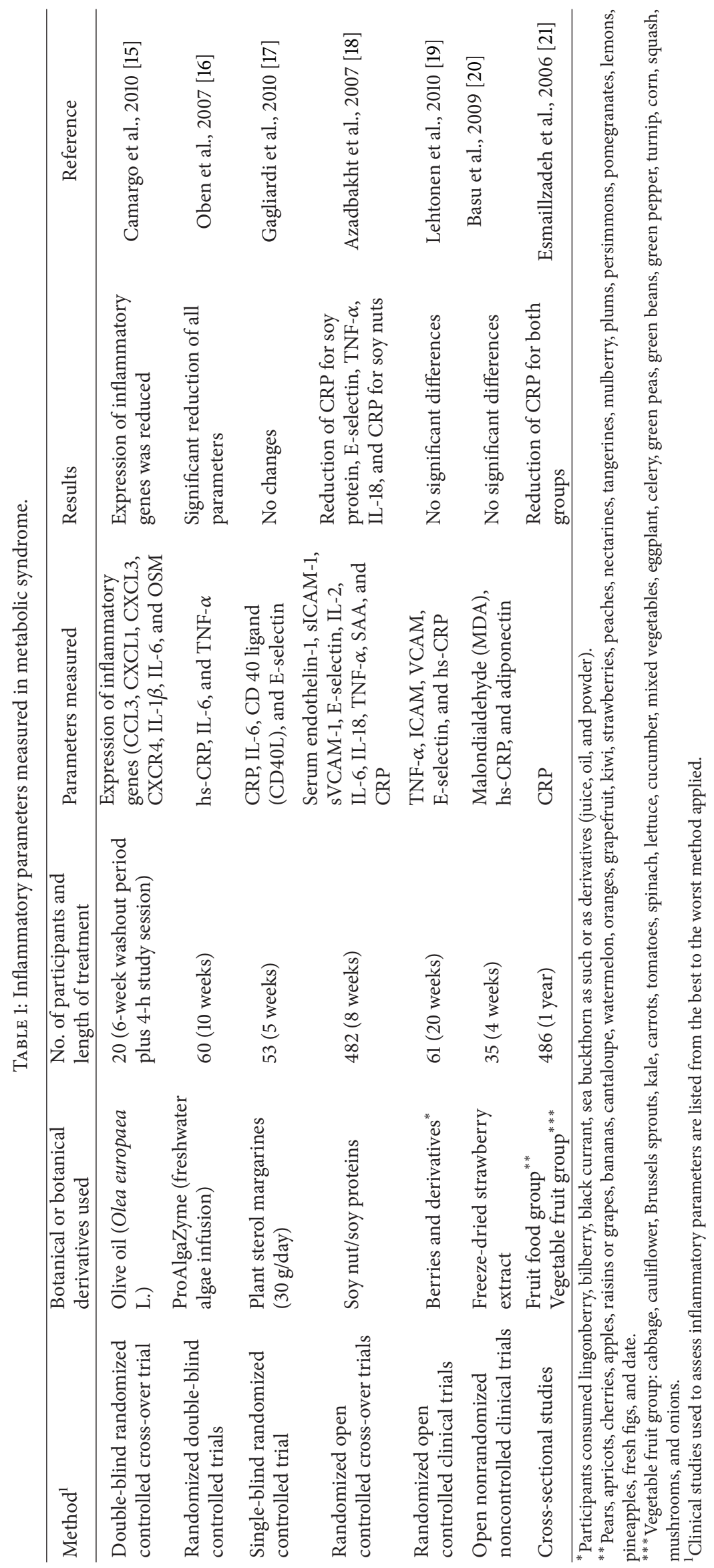


PFS treatment depend on the plant used and on the bioavailability of the active compounds and the method conditions, parameters that change in a short period of time can be selected as useful biomarkers of the anti-inflammatory properties of PFS. In patients affected by MS (Table 1), CRP levels decreased in three of the seven studies measuring it: a randomized open controlled cross-over study involving 482 patients for eight weeks [18], a 10-week randomized doubleblind controlled clinical trial involving 60 patients [16], and a 1-year cross-sectional study involving 486 patients [21].

On the contrary, the other four of these studies [17, 19, 20,23 ] reported no changes in CRP values. This discrepancy could be due to the nature of the botanicals used, the bioavailability of active compounds, the number of patients enrolled in the study, the type of study, and/or the laboratory methods for biomarker measurement. A larger number of studies is necessary to assess if CRP is a suitable parameter to evaluate the decrease of inflammatory status in MS after treatment with PFS.

E-selectin levels were evaluated in four studies [17-19, 23], but a decrease was recorded in only one [18], which rules out this biomarker as suitable for the purpose. TNF- $\alpha$ levels were evaluated in three studies $[16,18,19]$, and two of them $[16,18]$ reported a significant reduction after treatment with soy proteins ( 8 weeks) and fresh algae infusion (10 weeks). No significant differences before and after treatment with berry derivatives were found [19]. IL-18 was evaluated in only one study [18], and no conclusions can be drawn. IL-6 levels: two studies [17, 23] reported no significant differences after 5 and 9 weeks; another three studies $[16,18,20]$ reported a significant reduction in both gene expression and serum after 8,10 , and 20 weeks.

We may conclude that TNF- $\alpha$ and IL- 6 may be suitable biomarkers to evaluate in a rather short time (6-10 weeks) the improvement of the inflammatory status in humans after PFS treatment, but careful consideration of the botanical formulation used in the study (i.e., kind of extract, occurrence of active principles, and their bioavailability) and the amount of PFS taken before should be given before ruling a proinflammatory biomarker in or out. Additional studies are needed before considering CRP and IL-18 as biomarkers modulated by PFS treatment, since the data occurring in the literature do not allow us to draw clear conclusions. The importance of IL-6, TNF- $\alpha$, and CRP as proinflammatory biomarkers is well documented. TNF- $\alpha$ is released by adipose tissue and is overexpressed in obesity, and TNF- $\alpha$ can modulate insulin resistance in a variety of clinical trials related to obesity [6, $15,24]$. CRP and IL- 6 are peripheral inflammatory markers, and their measurement improves the prediction of the risk of cardiovascular events [24].

\subsection{Diabetes Studies}

3.2.1. In Vitro Methods. Table 2 reports the in vitro methods applied to investigate inflammatory markers in diabetes. The method mostly used for the assessment of beneficial effects of botanicals/plant food supplements on diabetes were based on quantitative PCR (qPCR), a real-time PCR coupled with reverse transcriptase. This is a very sensitive and specific method for analyzing mRNA levels as a marker of gene expression. Uemura et al. [25] reported the use of SYBR Green assay, while Chuang et al. [26, 27] and Cao et al. [28] reported the use of the highly specific and sensitiveTaQman assay. Other frequently used methods are ELISA assay, immunoblotting, and transfections, in a variety of cell cultures, with a plasmid containing the luciferase reporter gene under the control of the NF- $\kappa \mathrm{B}$ responsive element $(\mathrm{pNF}-\kappa \mathrm{B}-\mathrm{luc})$; the latter method is widely used for in vitro assays because it assesses events upstream of the inflammatory cascade which activates the NF- $\kappa \mathrm{B}$ pathway.

In the luciferase transfection assay only, Chuang et al. $[26,27]$ used primary cultures (from humans or animals) in their experiments; they are considered the most predictive, as primary cells retain the characteristics of the starting tissue. However, the isolation of appropriate cells from primary cultures can be difficult as the cell population is heterogeneous. Moreover, primary cultures have a limited life. Considering the challenges associated with modelling a chronic disease such as diabetes, it is encouraging to see that several in vitro methods for investigating inflammation have been developed, but few of them have been applied to the evaluation of PFS benefit assessment. In this sense, it would be interesting to develop methods to evaluate the following inflammatory events:

(1) nuclear translocation of NF- $\kappa \mathrm{B}$ factor in the nucleus (usually evaluated by ELISA test) and the consequent transcription of inflammatory genes and the expression of adhesion molecules on endothelium, leading to the vascular complications of diabetes;

(2) expression of adhesion molecules (i.e., sICAM-1, sICAM-2 and sVCAM-1, and E-selectin), which are typically overexpressed in diabetes and in cardiovascular disease [38]. Adhesion molecule expression is usually measured by RT-PCR (mRNA levels) and ELISA (protein expression on the cell surface);

(3) evaluation of metalloproteinase-9 (MMP-9) secretion and gene expression. MMP-9 is induced by hyperglycemia and accelerates some diabetic complications such as retinopathy [39]. MMP-9 gene expression is usually measured by RT-PCR, secretion and enzymatic activities by zymography or western blotting;

(4) evaluation of monocyte-macrophage chemotaxis in the endothelium. Indeed, monocytes and macrophages play a role in accelerating diabetes and in the development of atherosclerosis. They express specific receptors for advanced glycation end products (AGEs), which are proteins or lipids that become nonenzymatically glycated and oxidized after contact with aldose sugars.

After the binding of AGEs and intracellular processing, monocytes/macrophages synthesize and secrete growthpromoting cytokines such as TNF- $\alpha$, interleukin 1, and insulin-like growth factor, responsible for vascular complications in diabetes [40]. Furthermore, circulating AGEs may interact with endothelial receptors, which leads to 


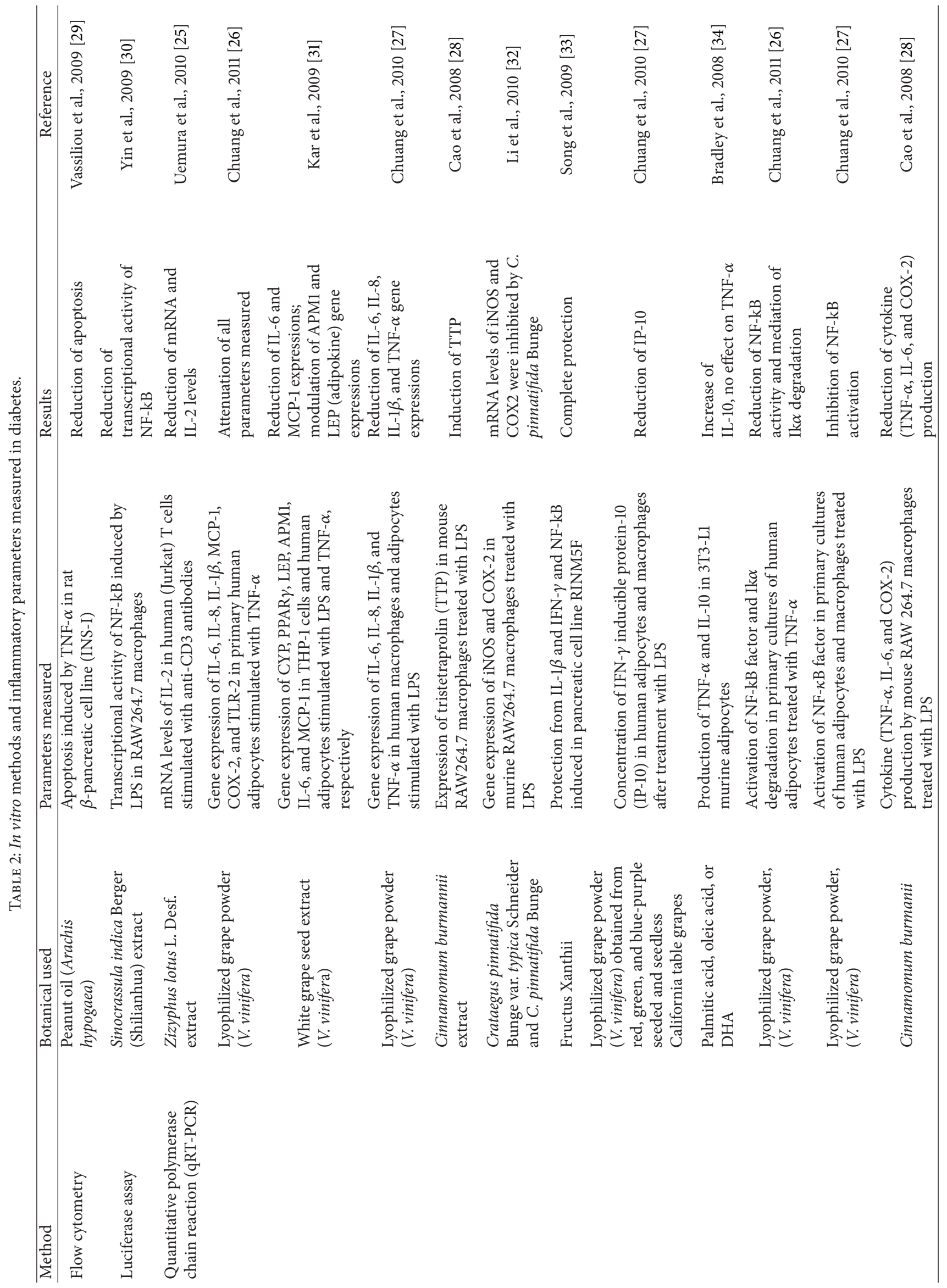




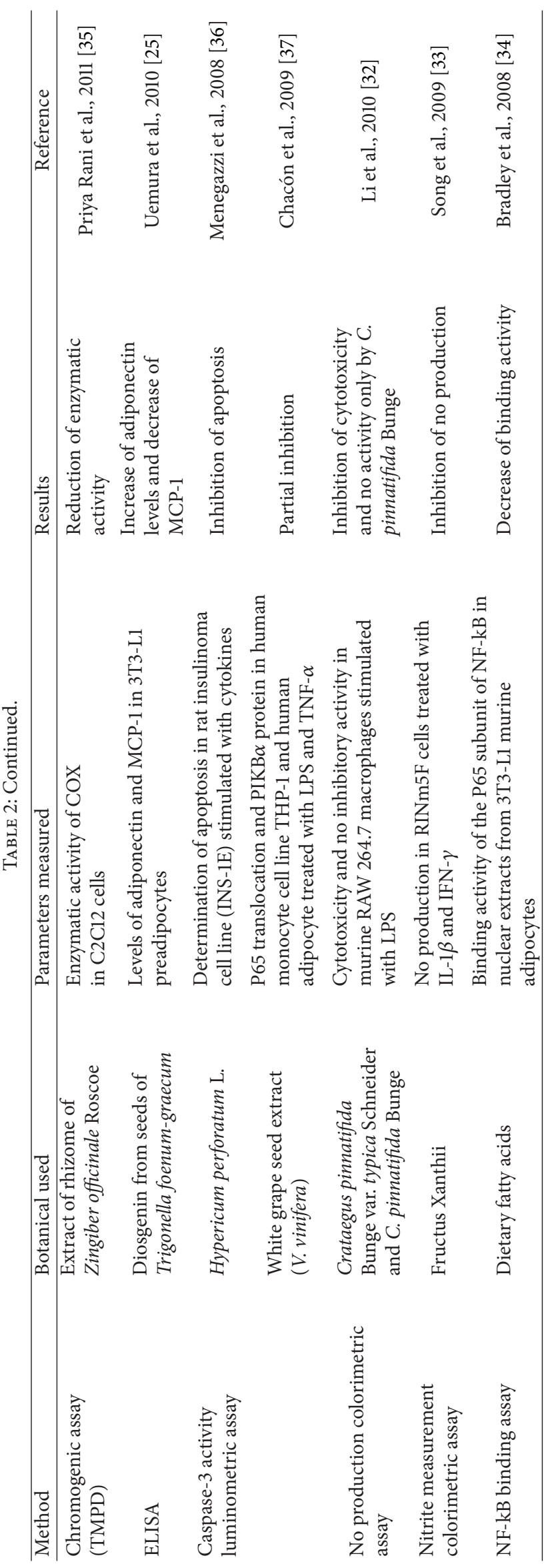




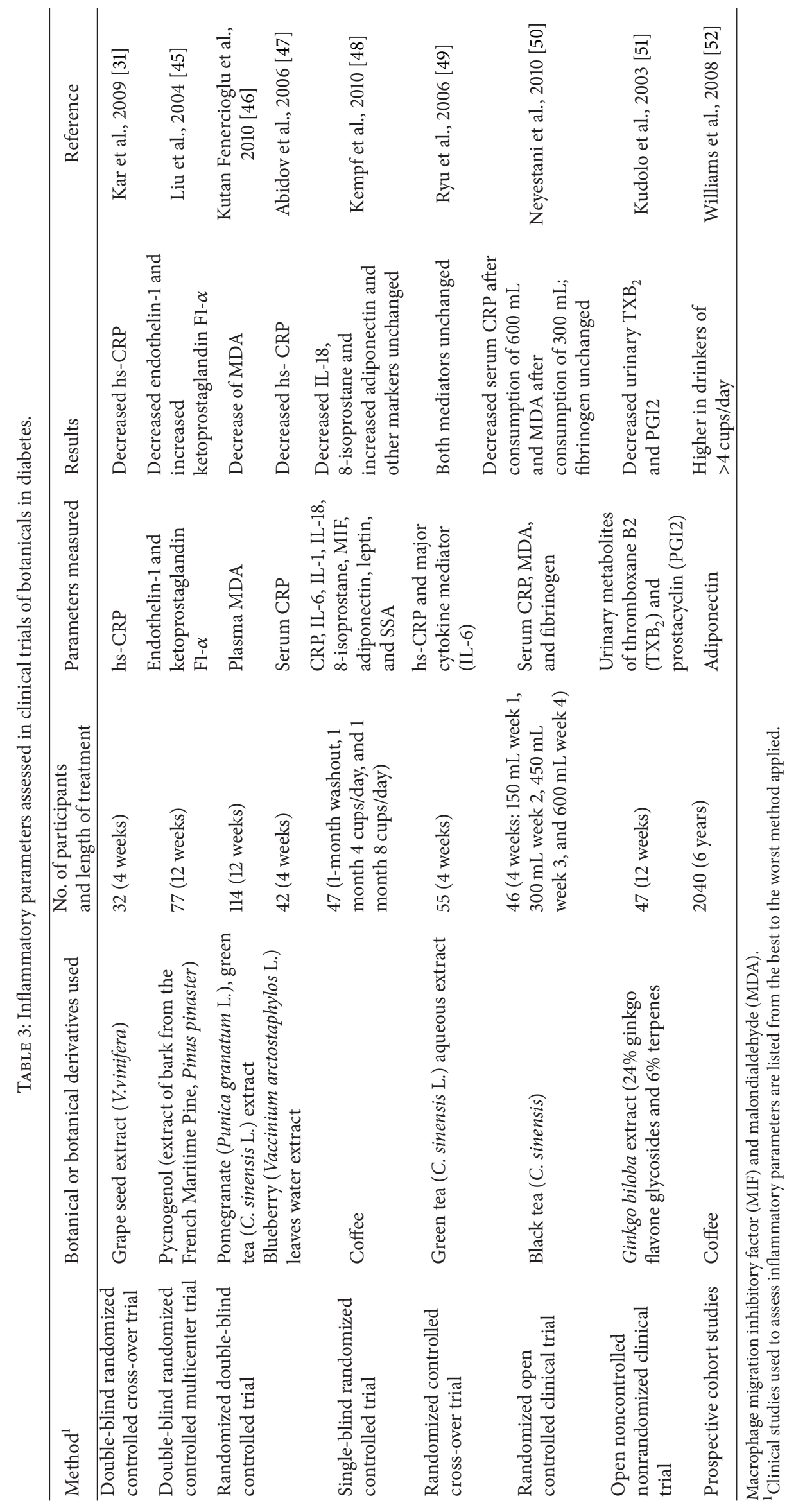


perturbation of cellular properties, such as upregulation of the transcription and the translocation of nuclear factor NF$\kappa \mathrm{B}$ [41]. Chemotaxis is generally measured under agarose $[42,43]$ or by chemotaxis assay, based on evaluation of cell migration through apposite filters after inflammatory stimuli have been placed in chambers (Boyden Chamber Assay) [44].

3.2.2. In Vivo Methods. Table 3 reports the in vivo methods developed for human trials.

The majority of clinical trials evaluating the effect of botanicals on inflammatory biomarkers in diabetic patients were randomized, double-blind randomized controlled trials and randomized, open and controlled studies. The lack of blinding is particularly critical when the treatment is applied versus placebo. In fact, the placebo effect is a major cause of bias due to patient or doctor awareness [22]. Several studies have indicated that nonrandomized trials are more likely to yield a positive result for a new treatment than for an established conventional one. In some of the clinical trials considered, randomization was not described in detail or was unsuitable. In two papers $[53,54]$, the number of subjects was limited. In some studies $[49,51,55]$, the dropout rate and the reasons for it were not critically discussed. The most often used methods for biomarker quantification were ELISA assay and HPLC analysis.

3.2.3. Inflammatory Biomarkers Affected by PFS in Diabetes. The parameters most frequently measured in clinical trials were as follows: $\mathrm{CRP}=\mathrm{MDA}>$ endothelin-1 = urinary thromboxane $\mathrm{B}_{2}$ metabolites $>$ IL-6, IL-18, TNF- $\alpha$, and $\mathrm{PGI}_{2}$, 8-isoprostane. Six studies [31, 47-50, 55] examined CRP levels; among them, three $[31,47,50]$ reported a significant reduction in serum CRP levels after 4 weeks of treatment, two studies reported no significant changes [48, 49], and one reported increased levels of CRP [55]. As for MS, further studies are mandatory before conclusions on the efficacy of in vivo CRP measurement in diabetes after PFS treatment can be drawn.

Serum levels of malondialdehyde (MDA) were measured in three studies $[46,50,56]$, and all reported a significant reduction after 4,8 , and 12 weeks of PFS consumption. MDA is an important biomarker of oxidative stress in diabetes as a consequence of persistent hyperglycemia and lipid peroxidation. These trials establish serum MDA measurement as appropriate for the purpose.

IL-6 levels were measured in three studies $[48,49,56]$ and one reported a significant reduction after PFS treatment [56]. IL-18 [48] and TNF- $\alpha$ [56] each were measured in only one study, and in both cases, a significant decrease was found. Nevertheless, further studies are needed to evaluate IL-6 and IL-18 as suitable inflammatory parameters. Endothelin1 , generally overexpressed in diabetes models [57], was found to decrease in two studies $[45,56]$, after 12 and 8 weeks of treatment, respectively.

Two studies showed a significant reduction of thromboxane $\mathrm{B}_{2}\left(\mathrm{TXB}_{2}\right)$ urinary metabolites after 4 and 12 weeks of treatment, respectively $[51,54]$. $\mathrm{PGI}_{2}[51]$ and 8 -isoprostane [48] were each evaluated only in one study; both decreased after PFS treatment.TXB $2, \mathrm{PGI}_{2}$, and 8-isoprostane are key proinflammatory biomarkers in diabetes. They are massively released in the wake of hypercoagulation and the vascular modifications that are typical for this disease. The paucity of studies in which these mediators were assayed in response to PFS treatment does not allow us to establish their usefulness in investigating the benefit of PFS treatment.

\section{Conclusions}

The aim of the present paper was to collect and critically discuss the existing experimental approaches used in vitro, in silico, and in vivo for benefit assessment of botanicals or plant food supplements (PFSs) in decreasing inflammation in MS-related diseases. PFS often consist of a complex mixture of compounds, which makes benefit assessment difficult and subject to interferences and false positives. The development of reliable methods for evaluating benefit assessment and their application and validation is therefore crucial.

No in silico methods were found in MS-related diseases. qRT-PCR was the in vitro method most widely applied for measuring the expression of inflammatory cytokines in diabetes, but no in vitro methods were found for testing PFS benefits in MS. Data from the in vivo studies (clinical trials) show that the inflammatory markers CRP, MDA, and $\mathrm{PGI}_{2}$ are likely to be useful in judging the efficacy of PFS treatment, although more studies are needed to validate this conclusion. In addition, two proinflammatory biomarkers, IL- 6 and TNF$\alpha$, were the only two parameters to change in both in vitro and in vivo systems, and these biomarkers should be carefully considered in future studies.

\section{Acknowledgments}

The writing of this paper was funded by the European Community's Seventh Framework Programme under Grant agreement no. 245199. It has been carried out within the PlantLIBRA project (website: http://www.plantlibra.eu). This paper does not necessarily reflect the Commission views of its future policy on this area. The fellowship of Elisa Colombo is partially funded by FSE, Regione Lombardia.

\section{References}

[1] A. Onat, "Metabolic syndrome: nature, therapeutic solutions and options," Expert Opinion on Pharmacotherapy, vol. 12, no. 12, pp. 1887-1900, 2011.

[2] J. C. Pickup and M. A. Crook, "Is type II diabetes mellitus a disease of the innate immune system?" Diabetologia, vol. 41, no. 10, pp. 1241-1248, 1998.

[3] J. M. Fernández-Real and J. C. Pickup, "Innate immunity, insulin resistance and type 2 diabetes," Trends in Endocrinology and Metabolism, vol. 19, no. 1, pp. 10-16, 2008.

[4] J. I. Cleeman, "Executive summary of the third report of the National Cholesterol Education Program (NCEP) expert panel on detection, evaluation, and treatment of high blood cholesterol in adults (adult treatment panel III)," JAMA, vol. 285, no. 19, pp. 2486-2497, 2001. 
[5] S. M. Grundy, J. I. Cleeman, C. N. Merz et al., "Implications of recent clinical trials for the National Cholesterol Education Program Adult Treatment Panel III Guidelines," Journal of the American College of Cardiology, vol. 44, no. 3, pp. 720-732, 2004.

[6] A. Festa, R. D’Agostino, G. Howard, L. Mykkänen, R. P. Tracy, and S. M. Haffner, "Chronic subclinical inflammation as part of the insulin resistance syndrome: the insulin resistance atherosclerosis study (IRAS)," Circulation, vol. 102, no. 1, pp. 4247, 2000.

[7] M. Trøseid, I. Seljeflot, and H. Arnesen, "The role of interleukin18 in the metabolic syndrome," Cardiovascular Diabetology, vol. 9, article 11, 2010.

[8] G. Solinas and M. Karin, "JNK1 and IKK $\beta$ : molecular links between obesity and metabolic dysfunction," The FASEB Journal, vol. 24, no. 8, pp. 2596-2611, 2010.

[9] T. Pischon, S. E. Hankinson, G. S. Hotamisligil, N. Rifai, W. C. Willett, and E. B. Rimm, "Habitual dietary intake of n-3 and n-6 fatty acids in relation to inflammatory markers among US men and women," Circulation, vol. 108, no. 2, pp. 155-160, 2003.

[10] G. Zhao, T. D. Etherton, K. R. Martin, S. G. West, P. J. Gillies, and P. M. Kris-Etherton, "Dietary $\alpha$-linolenic acid reduces inflammatory and lipid cardiovascular risk factors in hypercholesterolemic men and women," Journal of Nutrition, vol. 134, no. 11, pp. 2991-2997, 2004.

[11] I. Abete, A. Astrup, J. A. Martínez, I. Thorsdottir, and M. A. Zulet, "Obesity and the metabolic syndrome: role of different dietary macronutrient distribution patterns and specific nutritional components on weight loss and maintenance," Nutrition Reviews, vol. 68, no. 4, pp. 214-231, 2010.

[12] F. Visioli, "Nutritional support in the pharmacological treatment of metabolic syndrome," European Journal of Pharmacology, vol. 668, supplement 1, pp. S43-S49, 2011.

[13] M. Dell'agli, C. Di Lorenzo, M. Badea, E. Sangiovanni, L. Dima, and P. Restani, "Plant food supplements with anti-inflammatory properties: a systematic review (I)," Critical Reviews in Food Science and Nutrition, vol. 53, no. 4, pp. 403-413, 2013.

[14] M. Dell'agli, C. Di Lorenzo, M. Badea et al., "Plant food supplements with anti-inflammatory properties: a systematic review (II)," Critical Reviews in Food Science and Nutrition, vol. 53, no. 4, pp. 403-413, 2013.

[15] A. Camargo, J. Ruano, J. M. Fernandez et al., "Gene expression changes in mononuclear cells in patients with metabolic syndrome after acute intake of phenol-rich virgin olive oil," $B M C$ Genomics, vol. 11, no. 1, article 253, 2010.

[16] J. Oben, E. Enonchong, D. Kuate et al., "The effects of ProAlgaZyme novel algae infusion on metabolic syndrome and markers of cardiovascular health," Lipids in Health and Disease, vol. 6, article 20, 2007.

[17] A. C. M. Gagliardi, R. C. Maranho, H. P. D. Sousa, E. J. Schaefer, and R. D. Santos, "Effects of margarines and butter consumption on lipid profiles, inflammation markers and lipid transfer to HDL particles in free-living subjects with the metabolic syndrome," European Journal of Clinical Nutrition, vol. 64, no. 10, pp. 1141-1149, 2010.

[18] L. Azadbakht, M. Kimiagar, Y. Mehrabi, A. Esmaillzadeh, F. B. Hu, and W. C. Willett, "Soy consumption, markers of inflammation, and endothelial function: a cross-over study in postmenopausal women with the metabolic syndrome," Diabetes Care, vol. 30, no. 4, pp. 967-973, 2007.

[19] H. M. Lehtonen, J. P. Suomela, R. Tahvonen et al., "Berry meals and risk factors associated with metabolic syndrome," European Journal of Clinical Nutrition, vol. 64, no. 6, pp. 614-621, 2010.
[20] A. Basu, M. Wilkinson, K. Penugonda, B. Simmons, N. M. Betts, and T. J. Lyons, "Freeze-dried strawberry powder improves lipid profile and lipid peroxidation in women with metabolic syndrome: baseline and post intervention effects," Nutrition Journal, vol. 8, no. 1, article 43, 2009.

[21] A. Esmaillzadeh, M. Kimiagar, Y. Mehrabi, L. Azadbakht, F. B. $\mathrm{Hu}$, and W. C. Willett, "Fruit and vegetable intakes, C-reactive protein, and the metabolic syndrome," American Journal of Clinical Nutrition, vol. 84, no. 6, pp. 1489-1497, 2006.

[22] A. R. Jadad, R. A. Moore, D. Carroll et al., "Assessing the quality of reports of randomized clinical trials: is blinding necessary?" Controlled Clinical Trials, vol. 17, no. 1, pp. 1-12, 1996.

[23] J. Plat, G. Brufau, G. M. Dallinga-Thie, M. Dasselaar, and R. P. Mensink, "A plant stanol yogurt drink alone or combined with a low-dose statin lowers serum triacylglycerol and nonHDL cholesterol in metabolic syndrome patients," Journal of Nutrition, vol. 139, no. 6, pp. 1143-1149, 2009.

[24] P. Dandona, A. Aljada, A. Chaudhuri, P. Mohanty, and R. Garg, "Metabolic syndrome: a comprehensive perspective based on interactions between obesity, diabetes, and inflammation," Circulation, vol. 111, no. 11, pp. 1448-1454, 2005.

[25] T. Uemura, S. Hirai, N. Mizoguchi et al., "Diosgenin present in fenugreek improves glucose metabolism by promoting adipocyte differentiation and inhibiting inflammation in adipose tissues," Molecular Nutrition and Food Research, vol. 54, no. 11, pp. 1596-1608, 2010.

[26] C. C. Chuang, A. Bumrungpert, A. Kennedy et al., "Grape powder extract attenuates tumor necrosis factor $\alpha$-mediated inflammation and insulin resistance in primary cultures of human adipocytes," Journal of Nutritional Biochemistry, vol. 22, no. 1, pp. 89-94, 2011.

[27] C. C. Chuang, K. Martinez, G. Xie et al., "Quercetin is equally or more effective than resveratrol in attenuating tumor necrosis factor- $\alpha$-mediated inflammation and insulin resistance in primary human adipocytes," American Journal of Clinical Nutrition, vol. 92, no. 6, pp. 1511-1521, 2010.

[28] H. Cao, J. F. Urban, and R. A. Anderson, "Cinnamon polyphenol extract affects immune responses by regulating anti- and proinflammatory and glucose transporter gene expression in mouse macrophages," Journal of Nutrition, vol. 138, no. 5, pp. 833-840, 2008.

[29] E. K. Vassiliou, A. Gonzalez, C. Garcia, J. H. Tadros, G. Chakraborty, and J. H. Toney, "Oleic acid and peanut oil high in oleic acid reverse the inhibitory effect of insulin production of the inflammatory cytokine TNF- both in vitro and in vivo systems," Lipids in Health and Disease, vol. 8, article 25, 2009.

[30] J. Yin, A. Zuberi, Z. Gao, D. Liu, Z. Liu, and J. Ye, "Shilianhua extract inhibits GSK-3 $\beta$ and promotes glucose metabolism," American Journal of Physiology, vol. 296, no. 6, pp. E1275-E1280, 2009.

[31] P. Kar, D. Laight, H. K. Rooprai, K. M. Shaw, and M. Cummings, "Effects of grape seed extract in Type 2 diabetic subjects at high cardiovascular risk: a double blind randomized placebo controlled trial examining metabolic markers, vascular tone, inflammation, oxidative stress and insulin sensitivity," Diabetic Medicine, vol. 26, no. 5, pp. 526-531, 2009.

[32] C. Li, H. J. Son, C. Huang, S. K. Lee, J. Lohakare, and M. H. Wang, "Comparison of Crataegus pinnatifida Bunge var. typica Schneider and C. pinnatifida Bunge fruits for antioxidant, anti$\alpha$-glucosidase, and anti-inflammatory activities," Food Science and Biotechnology, vol. 19, no. 3, pp. 769-775, 2010. 
[33] M. Y. Song, E. K. Kim, H. J. Lee et al., "Fructus Xanthii extract protects against cytokine-induced damage in pancreatic $\beta$-cells through suppression of NF- $\kappa$ B activation," International Journal of Molecular Medicine, vol. 23, no. 4, pp. 547-553, 2009.

[34] R. L. Bradley, F. M. Fisher, and E. Maratos-Flier, "Dietary fatty acids differentially regulate production of TNF- $\alpha$ and IL-10 by murine 3T3-L1 adipocytes," Obesity, vol. 16, no. 5, pp. 938-944, 2008.

[35] M. Priya Rani, K. P. Padmakumari, B. Sankarikutty, O. Lijo Cherian, V. M. Nisha, and K. G. Raghu, "Inhibitory potential of ginger extracts against enzymes linked to type 2 diabetes, inflammation and induced oxidative stress," International Journal of Food Sciences and Nutrition, vol. 62, no. 2, pp. 106-110, 2011.

[36] M. Menegazzi, M. Novelli, P. Beffy et al., "Protective effects of St. John's wort extract and its component hyperforin against cytokine-induced cytotoxicity in a pancreatic $\beta$-cell line," International Journal of Biochemistry and Cell Biology, vol. 40, no. 8, pp. 1509-1521, 2008.

[37] M. R. Chacón, V. Ceperuelo-Mallafré, E. Maymó-Masip et al., "Grape-seed procyanidins modulate inflammation on human differentiated adipocytes in vitro," Cytokine, vol. 47, no. 2, pp. 137-142, 2009.

[38] C. Urso, E. Hopps, and G. Caimi, "Adhesion molecules and diabetes mellitus," La Clinica Terapeutica, vol. 161, no. 1, pp. el7e24, 2010.

[39] R. A. Kowluru, "Role of matrix metalloproteinase-9 in the development of diabetic retinopathy and its regulation by $\mathrm{H}-$ Ras," Investigative Ophthalmology and Visual Science, vol. 51, no. 8, pp. 4320-4326, 2010.

[40] M. Kirstein, J. Brett, S. Radoff, S. Ogawa, D. Stern, and H. Vlassara, "Advanced protein glycosylation induces transendothelial human monocyte chemotaxis and secretion of platelet-derived growth factor: role in vascular disease of diabetes and aging," Proceedings of the National Academy of Sciences of the United States of America, vol. 87, no. 22, pp. 9010-9014, 1990.

[41] A. Goldin, J. A. Beckman, A. M. Schmidt, and M. A. Creager, "Advanced glycation end products: sparking the development of diabetic vascular injury," Circulation, vol. 114, no. 6, pp. 597605, 2006.

[42] L. S. Martin, T. J. Spira, S. L. Orloff, and R. C. Holman, "Comparison of methods for assessing chemotaxis of monocytes and polymorphonuclear leukocytes isolated from patients with AIDS or AIDS-related conditions," Journal of Leukocyte Biology, vol. 44, no. 5, pp. 361-366, 1988.

[43] R. D. Nelson, P. G. Quie, and R. L. Simmons, "Chemotaxis under agarose: a new and simple method for measuring chemotaxis and spontaneous migration of human polymorphonuclear leukocytes and monocytes," The Journal of Immunology, vol. 115, no. 6, pp. 1650-1656, 1975.

[44] R. Snyderman, L. C. Altman, M. S. Hausman, and S. E. Mergenhagen, "Human mononuclear leukocyte chemotaxis: a quantitative assay for humoral and cellular chemotactic factors," The Journal of Immunology, vol. 108, no. 3, pp. 857-860, 1972.

[45] X. Liu, J. Wei, F. Tan, S. Zhou, G. Würthwein, and P. Rohdewald, "Antidiabetic effect of Pycnogenol French maritime pine bark extract in patients with diabetes type II," Life Sciences, vol. 75, no. 21, pp. 2505-2513, 2004.

[46] A. Kutan Fenercioglu, T. Saler, E. Genc, H. Sabuncu, and Y. Altuntas, "The effects of polyphenol-containing antioxidants on oxidative stress and lipid peroxidation in Type 2 diabetes mellitus without complications," Journal of Endocrinological Investigation, vol. 33, no. 2, pp. 118-124, 2010.

[47] M. Abidov, A. Ramazanov, M. Jimenez Del Rio, and I. Chkhikvishvili, "Effect of Blueberin on fasting glucose, Creactive protein and plasma aminotransferases, in female volunteers with diabetes type 2: double-blind, placebo controlled clinical study," Georgian Medical News, no. 141, pp. 66-72, 2006.

[48] K. Kempf, C. Herder, I. Erlund et al., "Effects of coffee consumption on subclinical inflammation and other risk factors for type 2 diabetes: a clinical trial," American Journal of Clinical Nutrition, vol. 91, no. 4, pp. 950-957, 2010.

[49] O. H. Ryu, J. Lee, K. W. Lee et al., "Effects of green tea consumption on inflammation, insulin resistance and pulse wave velocity in type 2 diabetes patients," Diabetes Research and Clinical Practice, vol. 71, no. 3, pp. 356-358, 2006.

[50] T. R. Neyestani, N. Shariatzade, A. Kalayi et al., "Regular daily intake of black tea improves oxidative stress biomarkers and decreases serum C-reactive protein levels in type 2 diabetic patients," Annals of Nutrition and Metabolism, vol. 57, no. 1, pp. 40-49, 2010.

[51] G. B. Kudolo, S. Dorsey, and J. Blodgett, "Effect of the ingestion of Ginkgo biloba extract on platelet aggregation and urinary prostanoid excretion in healthy and Type 2 diabetic subjects," Thrombosis Research, vol. 108, no. 2-3, pp. 151-160, 2002.

[52] C. J. Williams, J. L. Fargnoli, J. J. Hwang et al., "Coffee consumption is associated with higher plasma adiponectin concentrations in women with or without type 2 diabetes: a prospective cohort study," Diabetes Care, vol. 31, no. 3, pp. 504507, 2008.

[53] L. Axelrod, J. Camuso, E. Williams, K. Kleinman, E. Briones, and D. Schoenfeld, "Effects of a small quantity of $\omega-3$ fatty acids on cardiovascular risk factors in NIDDM: a randomized, prospective, double-blind, controlled study," Diabetes Care, vol. 17, no. 1, pp. 37-44, 1994.

[54] R. Takahashi, J. Inoue, H. Ito, and H. Hibino, "Evening primrose oil and fish oil in non-insulin-dependent-diabetes," Prostaglandins Leukotrienes and Essential Fatty Acids, vol. 49, no. 2, pp. 569-571, 1993.

[55] Y. Fukino, M. Shimbo, N. Aoki, T. Okubo, and H. Iso, "Randomized controlled trial for an effect of green tea consumption on insulin resistance and inflammation markers," Journal of Nutritional Science and Vitaminology, vol. 51, no. 5, pp. 335-342, 2005.

[56] P. Usharani, A. A. Mateen, M. U. R. Naidu, Y. S. N. Raju, and N. Chandra, "Effect of NCB-02, atorvastatin and placebo on endothelial function, oxidative stress and inflammatory markers in patients with type 2 diabetes mellitus: a randomized, parallel-group, placebo-controlled, 8-week study," Drugs in $R$ and D, vol. 9, no. 4, pp. 243-250, 2008.

[57] A. G. Minchenko, M. J. Stevens, L. White et al., "Diabetesinduced overexpression of endothelin-1 and endothelin receptors in the rat renal cortex is mediated via poly(ADP-ribose) polymerase activation," The FASEB Journal, vol. 17, no. 11, pp. 1514-1516, 2003. 


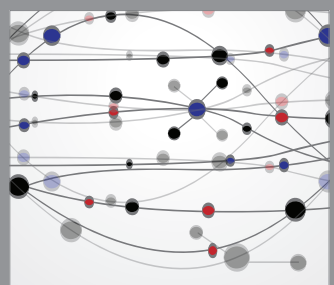

The Scientific World Journal
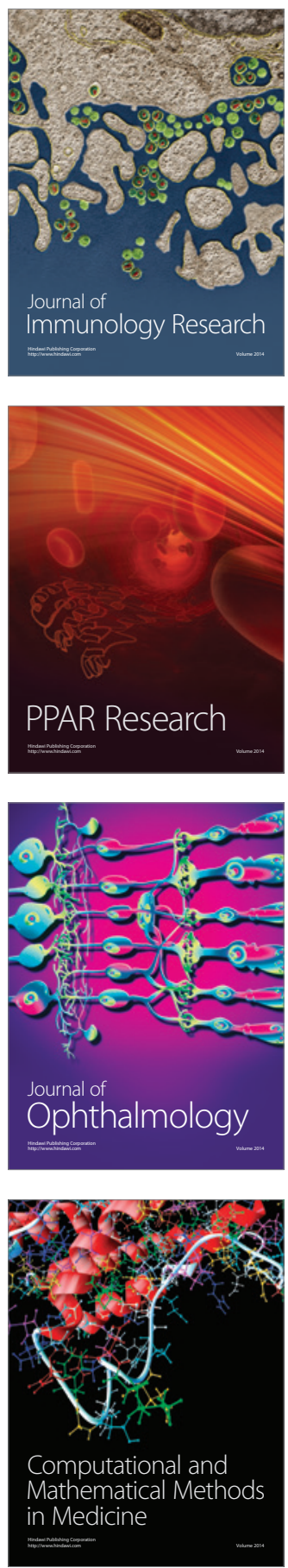

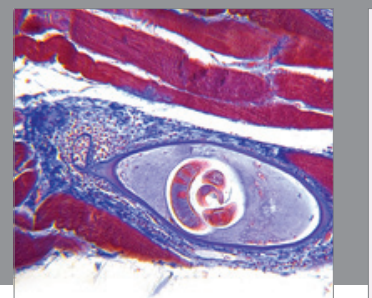

Gastroenterology

Research and Practice
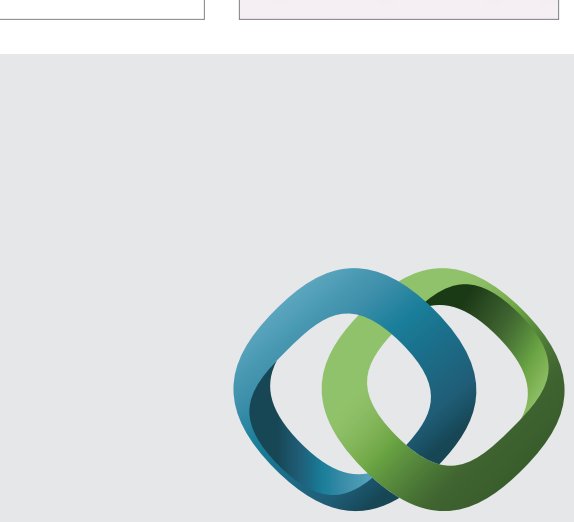

\section{Hindawi}

Submit your manuscripts at

http://www.hindawi.com
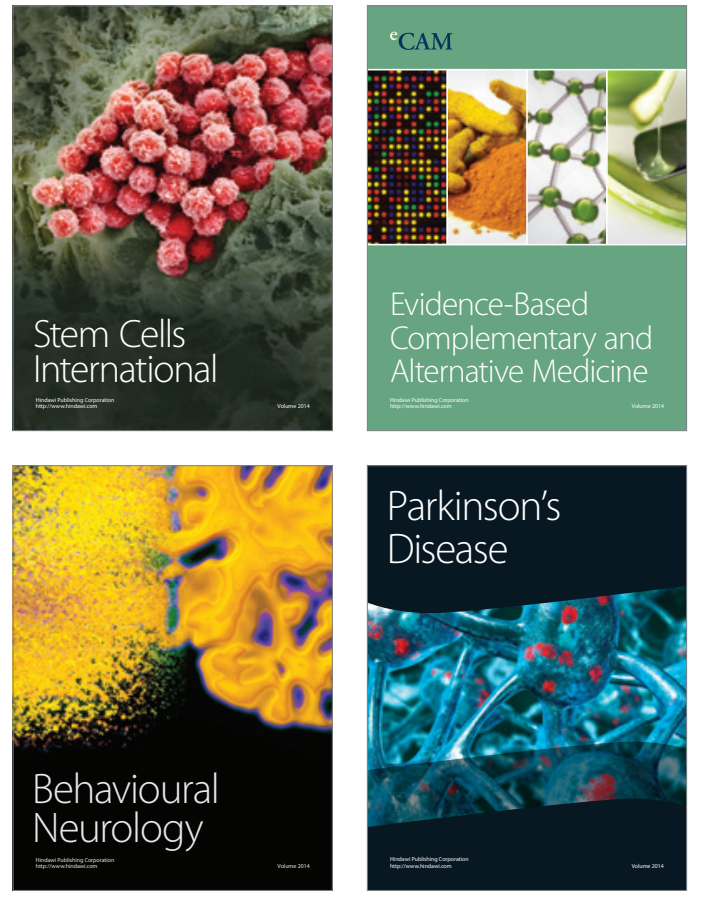
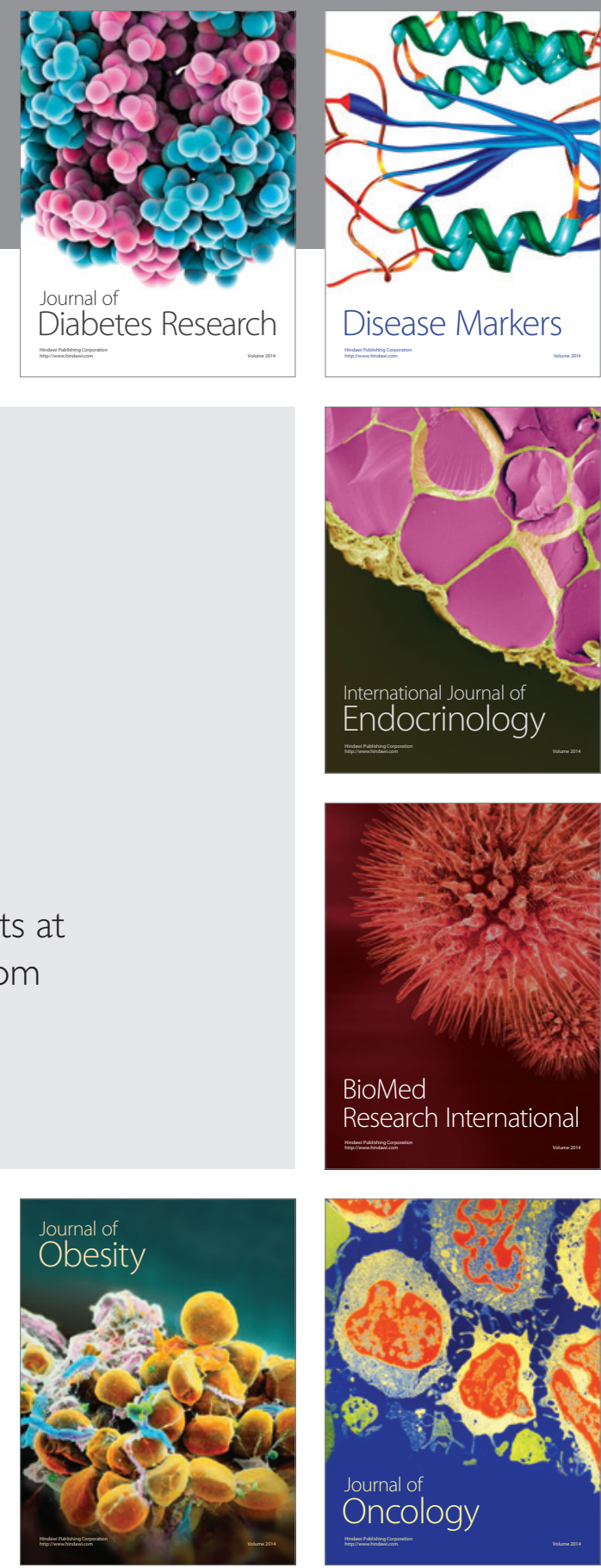

Disease Markers
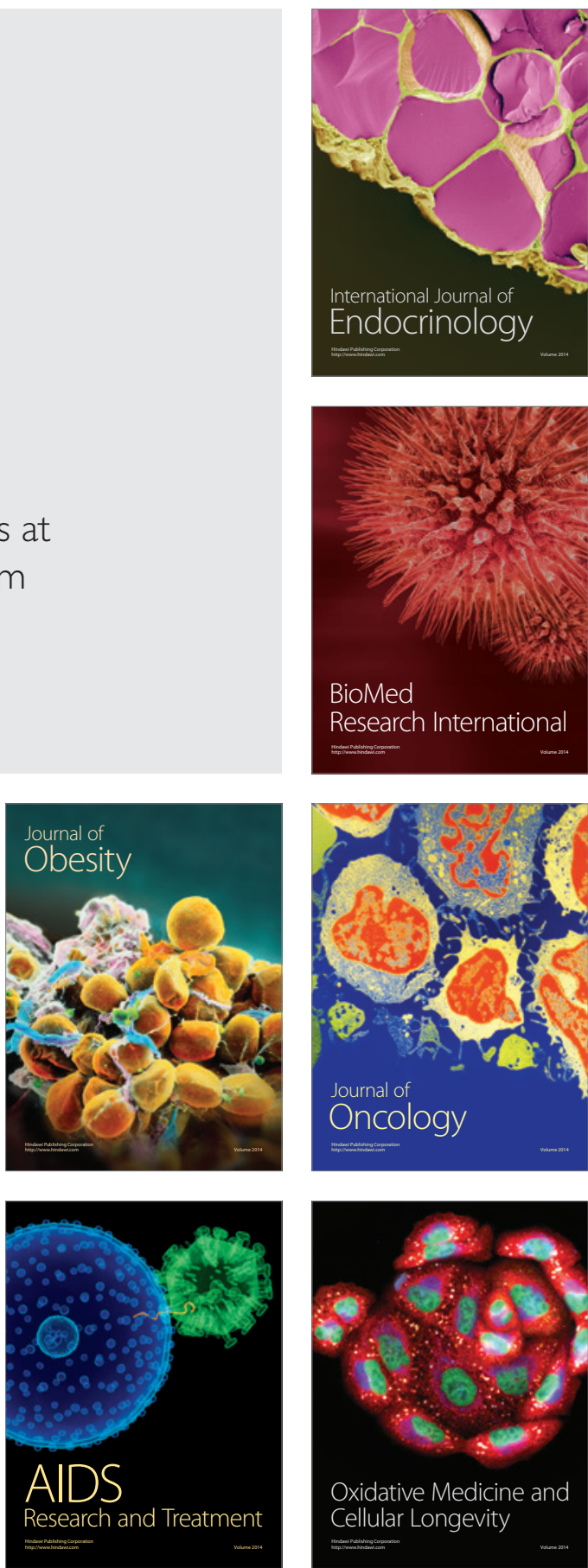